\title{
Transport Rate of Gaseous Phosphorus into Solid Iron under Reducing Atmosphere
}

\author{
Minoru SASABE, Yoshinari KIYOSAWA1), Michio OTSUKA, Katsuhiko TANAKA and Mitsuru TATE
}

Department of Metallurgical Engineering, Chiba Institute of Technology, Tsudanuma, Narashino, Chiba-ken, 275 Japan. 1 1) Graduate School, Chiba Institute of Technology. Now at Oyama Works, Tokyo Tekko Co., Ltd., Yokokura Shin-den, Oyama, Tochigi-ken, 323 Japan.

(Received on October 5, 1990; accepted in the final form on January 25, 1991)

\begin{abstract}
Transport rate of gaseous phosphorus into solid iron under reducing atmosphere was observed by a gravimetric procedure to obtain fundamental data to discuss transport mechanism of phosphorus into reduced iron in a blast furnace. A kind of killed and rolled steel sheet and non worked electrolytic iron sheet were used as samples. Partial pressure of phosphorus vapor was controlled by using equilibrium relation between $P_{\mathrm{P} 2}, \mathrm{Ca}_{3}\left(\mathrm{PO}_{4}\right)_{2}, \mathrm{SiO}_{2}, \mathrm{C}, \mathrm{CaSiO}_{3}$ and $\mathrm{CO}$. Phosphorus partial pressure and temperature were every one order between $4 \times 10^{-6}$ and $4 \times 10^{-2}$ atm, and every $50^{\circ} \mathrm{C}$ between 900 and $1050^{\circ} \mathrm{C}$, respectively.

Experimental results are as follows:

(1) Transport rate obeyed a parabolic rate law.

(2) Relations between the rate constants of the parabolic rate law, $k$, the phosphorus partial pressure, $P_{\mathrm{P} 2}$, and the temperature, $T$, are as follows:

In the case of killed steel, $\log k=0.14 \log P_{\mathrm{P} 2}+5.68-15.6 \times 10^{3} / T$

In the case of the electrolytic iron, $\log k=0.14 \log P_{\mathrm{P} 2}+4.94-15.6 \times 10^{3} / T$

(3) Reaction product on a surface of solid iron was $\mathrm{Fe}_{2} P$.
\end{abstract}

KEY WORDS: ironmaking; solid iron; phosphorus; vapor pressure; transport rate; temperature; diffusion.

\section{Introduction}

There are many studies on dephosphorization phenomena of molten steel since Herty's one. ${ }^{1)}$ It is believed that phosphorus absorption reaction of reduced iron in a blast furnace is a reaction between molten slag and metal phases. On the other hand, Oelsen and Maetz $^{2)}$ and Jon et al.$^{3)}$ were proposed that it was a reaction between gas and solid metal phases, however there was little experimental study on the reaction between solid iron and phosphorus vapor. A study by Sasa and $\mathrm{Cho}^{4}$ ) on an effect of vapor pressure of phosphorus on reduction rate of iron oxide is only one example of the study on the reaction between solid iron and gaseous phosphorus.

It is an object of the present study to obtain fundamental data in order to discuss transport mechanism of phosphorus into reduced iron in a blast furnace.

\section{Experimental Procedure}

Transport rate of gaseous phosphorus into solid iron is measured by two gravimetric procedures using batch as well as continuous methods. The transport rate of phosphorus into killed steel sheet with higher purity of which composition was shown in Table 1 was measured by a batch method to observe weight gain during apparently longer reaction time. In the case of the batch method, a steel sheet of $20 \mathrm{~mm}$ in length, $15 \mathrm{~mm}$ in width and $1 \mathrm{~mm}$ in thickness was hung in a reaction tube made of silica, heated in $\mathrm{N}_{2}$ atmosphere and then reacted with phosphorus vapor. The weight gain of the sample was measured by a balance after cooling.

A sample to observe the weight gain during apparent shorter reaction time was non-worked electrolytic iron sheet of which composition was shown in Table 2. The sheet $25 \mathrm{~mm}$ in length, $8 \mathrm{~mm}$ in width and $2.5 \mathrm{~mm}$ in thickness, was hung in a reaction tube made of silica of $18 \mathrm{~mm}$ in inner diameter. Weight change was continuously measured by thermobalance.

Vapor of phosphorus was liberated in a silica tube located beside the reaction tube above mentioned. The silica tube to liberate phosphorus vapor and the reaction tube were connected each other with a glass tube heated by nichrome wire wound on the surface of the glass tube. The reaction tube and the tube to liberate phosphorus vapor were heated by furnaces with silicon carbide heater.

Mixture of powder of $\mathrm{Ca}_{3}\left(\mathrm{PO}_{4}\right)_{2}, \mathrm{SiO}_{2}, \mathrm{C}$ and

Table 1. Chemical composition of killed steel sheet. (mass\%)

\begin{tabular}{cccccccccccc}
\hline $\mathrm{C}$ & $\mathrm{Si}$ & $\mathrm{Mn}$ & $\mathrm{P}$ & $\mathrm{S}$ & $\mathrm{Cu}$ & $\mathrm{Ni}$ & $\mathrm{Cr}$ & $\mathrm{Mo}$ & $\mathrm{Ti}$ & $\mathrm{V}$ & $\mathrm{Nb}$ \\
\hline 0.003 & 0.016 & 0.180 & 0.008 & 0.005 & 0.012 & 0.027 & 0.012 & 0.006 & 0.061 & 0.004 & 0.004 \\
\hline
\end{tabular}


$\mathrm{CaSiO}_{3}$ were filled in the silica tube to liberate phosphorus vapor. Phosphorus vapor with accurate partial pressure was liberated when $\mathrm{CO}$ and $\mathrm{N}_{2}$ controlled accurately by a mass flow controller were passed through the mixture layer of powder of which temperature was accurately controlled, e.g., phosphorus vapor pressure was determined by an equilibrium relation expressed by the following equation.

$$
\mathrm{Ca}_{3}\left(\mathrm{PO}_{4}\right)_{2}+3 \mathrm{SiO}_{2}+5 \mathrm{C}=3 \mathrm{CaSiO}_{3}+5 \mathrm{CO}+\mathrm{P}_{2}
$$

Flow rates of $\mathrm{CO}$ and $\mathrm{N}_{2}$ were 100 and $400 \mathrm{~m} l / \mathrm{min}$, respectively. The vapor pressure of phosphorus was controlled with no flow rate change but temperature change. Sequence of the gas how in a reaction apparatus is shown in Fig. 1.

A specimen before reaction was polished by \#1200 sand paper, dipped in dilute solution of $\mathrm{HCl}$ and then in ethyl alcohol, finally dried by blast of air. While the specimen was heated to reaction temperature, 500 $\mathrm{m} l / \mathrm{min}$ of dried and deoxidized $\mathrm{N}_{2}$ instead of the $\mathrm{CO}$ and $\mathrm{N}_{2}$ mixture was supplied to the reaction tube as well as to the tube to liberate phosphorus vapor. When temperature reached to experimental one, $\mathrm{N}_{2}$ gas supplied to the both tubes was stopped and mixture of $\mathrm{CO}$ and $\mathrm{N}_{2}$ was introduced into the tube to liberate phosphorus vapor. Exhaust gas with phosphorus vapor from the tube was introduced into the

Table 2. Chemical composition of electrolytic iron. (mass ppm)

\begin{tabular}{ccccccccccc}
\hline $\mathrm{G}$ & $\mathrm{P}$ & $\mathrm{S}$ & $\mathrm{Si}$ & $\mathrm{Mn}$ & $\mathrm{Cu}$ & $\mathrm{As}$ & $\mathrm{B}$ & $\mathrm{N}$ & $\mathrm{O}$ & $\mathrm{H}$ \\
\hline 16 & 1 & 6 & $\leqq 10$ & 1 & 3 & 3 & 1 & 9 & 26 & 2 \\
\hline \hline $\mathrm{Al}$ & $\mathrm{Bi}$ & $\mathrm{Cd}$ & $\mathrm{Co}$ & $\mathrm{Cr}$ & $\mathrm{Ni}$ & $\mathrm{Pb}$ & $\mathrm{Sb}$ & $\mathrm{Sn}$ & $\mathrm{Zn}$ \\
\hline 1 & $\leqq 1$ & $\leqq 1$ & 2 & 1 & 1 & 1 & 1 & 3 & 4
\end{tabular}

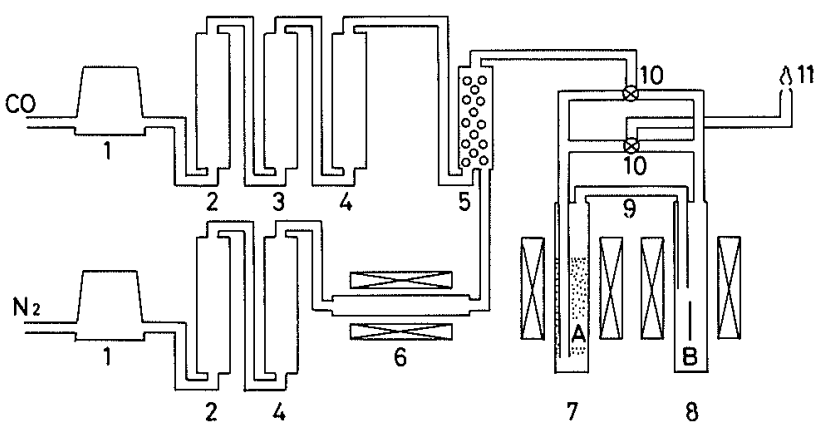

1: Mass flow controller

2: Silica gel

3: Soda lime

4: Magnesium perchlorate

5: Gas mixing tube with glass beads

6: Deoxidized furnace with metallic magnesium

7: Reaction tube made of silica

8: Apparatus for liberating phosphorus vapor made of silica

9: Glass tube with heater

10: Stopcock with three ways

11: Exhaust tube

A: Phosphorus source B: Specimen

Fig. 1. Schematic diagram of gas flow in the experimental apparatus. reaction tube.

The specimen after reaction was cooled in $\mathrm{N}_{2}$ atmosphere with flow rate $500 \mathrm{ml} / \mathrm{min}$. After cooling the specimen by the batch method was measured weight gain. Cross section of some specimens was observed by EPMA and SEM.

Reaction temperature was every $50^{\circ} \mathrm{G}$ between 900 to $1050^{\circ} \mathrm{C}$. The vapor pressure of phosphorus was every one order between $10^{-6}$ to $10^{-2} \mathrm{~atm}$. Relation between phosphorus vapor pressure and temperature of the tube liberating phosphorus vapor calculated on the basis of thermochemical data ${ }^{5}$ is shown in Table 3. The temperature was able to control by PID controller within $\pm 3^{\circ} \mathrm{C}$.

\section{Experimental Results}

\subsection{Control of Phosphorus Partial Pressure}

Partial pressure of phosphorus in the reaction tube was doublely ascertained by the following two experimental procedures, i.e., a condensation of phosphorus vapor in a cold trap and a measurement of weight loss of phosphorus source. The cold trap was consisted of an aluminum tube filled with small sized aluminum chips very much. It was cooled by ethyl alcohol with dry ice.

Partial pressure of $P_{\mathrm{P}_{2}}$ as phosphorus gas was estimated on the basis of the weight changes of the cold trap and the phosphorus source, the gas flow rate and the reaction time. Partial pressure of $P_{\mathrm{P}_{4}}$ was neglected because of one or two orders smaller than that of $P_{\mathrm{P}_{2}}{ }^{6}$ ) On the basis of the experimental results it has been ascertained that the partial pressure of $P_{\mathrm{P}_{2}}$ was almost the same as that estimated by thermochemical data ${ }^{5)}$ as shown in Fig. 2.

Table 3. Relation between furnace temperature and phosphorus partial pressure.

\begin{tabular}{cccccc}
\hline Temp. $\left({ }^{\circ} \mathrm{C}\right)$ & 1127 & 1152 & 1177 & 1202 & 1227 \\
\hline Press. (atm) & $4 \times 10^{-6}$ & $4 \times 10^{-5}$ & $4 \times 10^{-4}$ & $4 \times 10^{-8}$ & $4 \times 10^{-2}$ \\
\hline
\end{tabular}

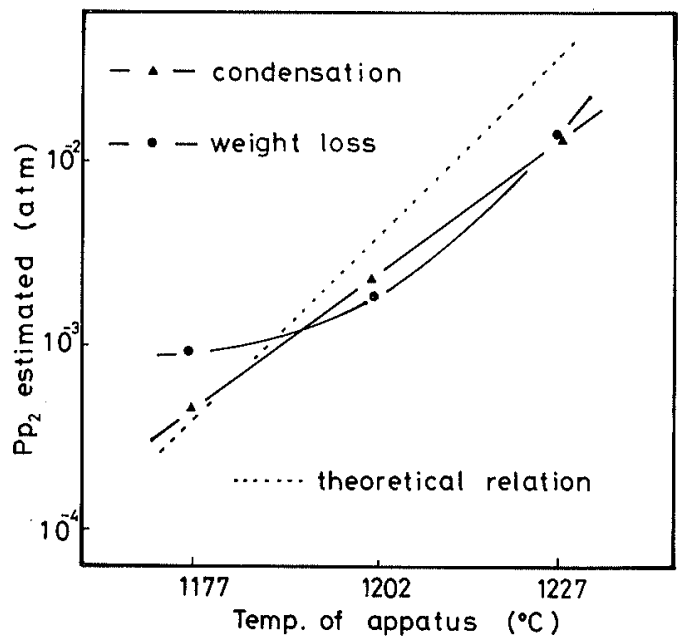

Fig. 2. Relation between temperature of the apparatus for liberating phosphorus vapor and partial pressure of phosphorus estimated on the basis of the condensation amount as well as weight loss. 
The phosphorus vapor was only controlled by temperature because it was experimentally ascertained that the partial pressure did not depend on ratio of $\mathrm{CO}$ to $\mathrm{N}_{2}$ introduced into the phosphorus source.

\subsection{Phosphorus Absorption Rate of Solid Iron}

Experimental results in the case of batch method using the steel sheet at $1000^{\circ} \mathrm{C}$ are shown in Fig. 3 as an example of experimental results by the batch method. The figure shows that the higher temperature and the higher phosphorus partial pressure, the higher reaction rate.

Experimental results in the case of the continuous method using electrolytic iron under $4 \times 10^{-3} \mathrm{~atm}$ of phosphorus partial pressure and at $1050^{\circ} \mathrm{C}$ are shown in Fig. 4 as an example of experimental results by the continuous method. Minus region on a time axis in the figure corresponds to a period of temperature rising of the specimen. No increase in weight during temperature rising can be observed. $\mathrm{N}_{2}$ atmosphere was changed to mixture of $\mathrm{N}_{2}$ and $\mathrm{CO}$ containing phosphorus vapor at time 0 . A rapid increase in weight of the specimen was observed immediately after the changing. It is estimated that the rapid

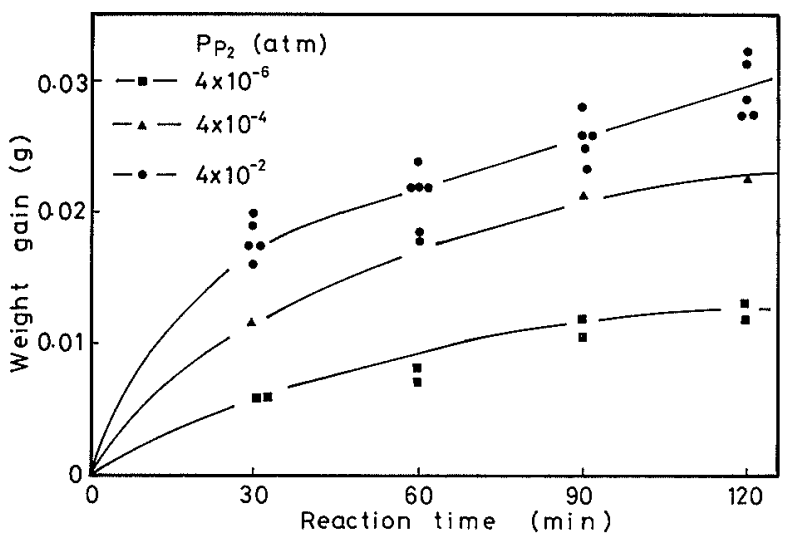

Fig. 3. Relation between weight gain of the sample and reaction time as a function of phosphorus partial pressure in the case of the killed steel at $1000^{\circ} \mathrm{C}$.

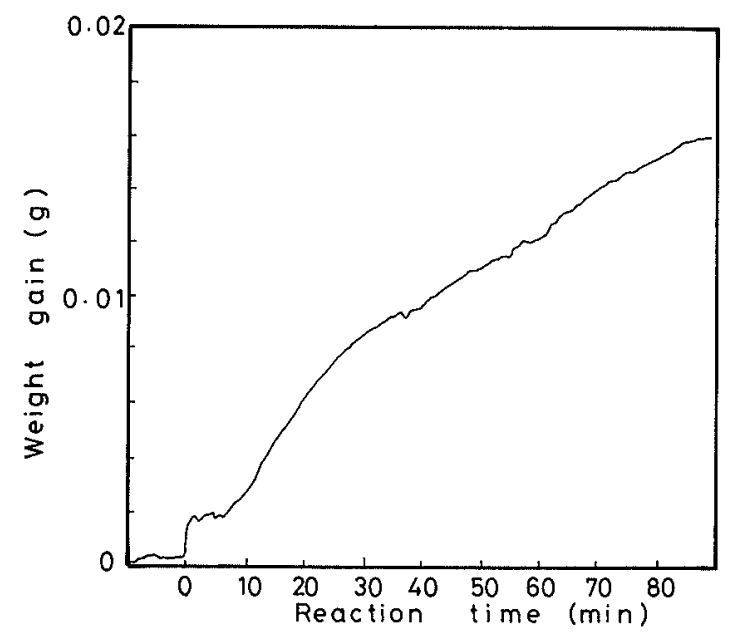

Fig. 4. Relation between weight gain of the sample and reaction time in the case of electrolytic iron under $4 \times$ $10^{-3} \mathrm{~atm}$ of phosphorus partial pressure and at $1050^{\circ} \mathrm{C}$. increase in weight corresponds to zero point shift of the thermoblance because the amount of the rapid weight change was different in each run.

After the zero point shift no weight change, i.e., an incubation period, was observed in $10 \mathrm{~min}$. It is estimated that the incubation period corresponds to an alternative time of gas in the reaction apparatus. After the incubation period the sample weight increases gradually. After the gradual weight change, stagnant and/or accelerative changes of weight were observed.

Relation between the gradual weight change and reaction time can be expressed as a parabolic rate law as shown in Figs. 5 to 7 . In Fig. 7 the incubation period are omitted because of avoidance of trouble-

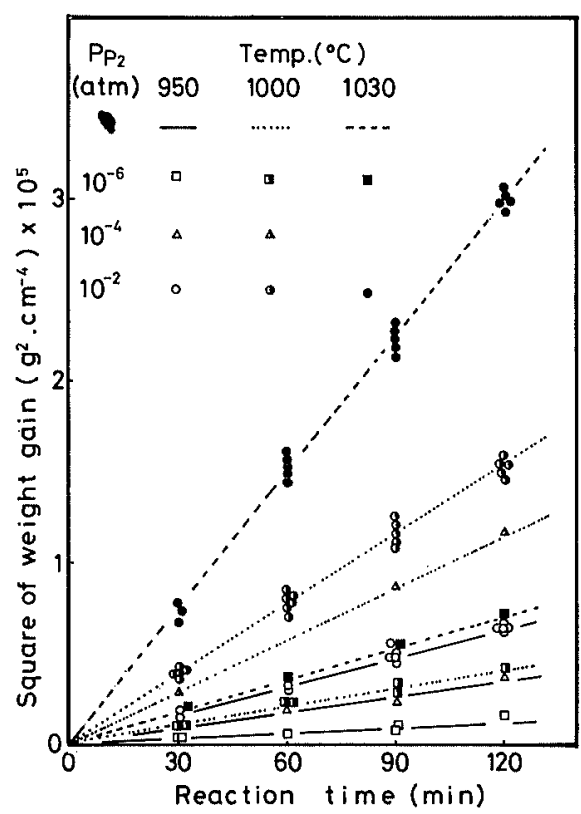

Fig. 5. Parabolic relation between weight gain of the sample and reaction time in the case of the killed steel.

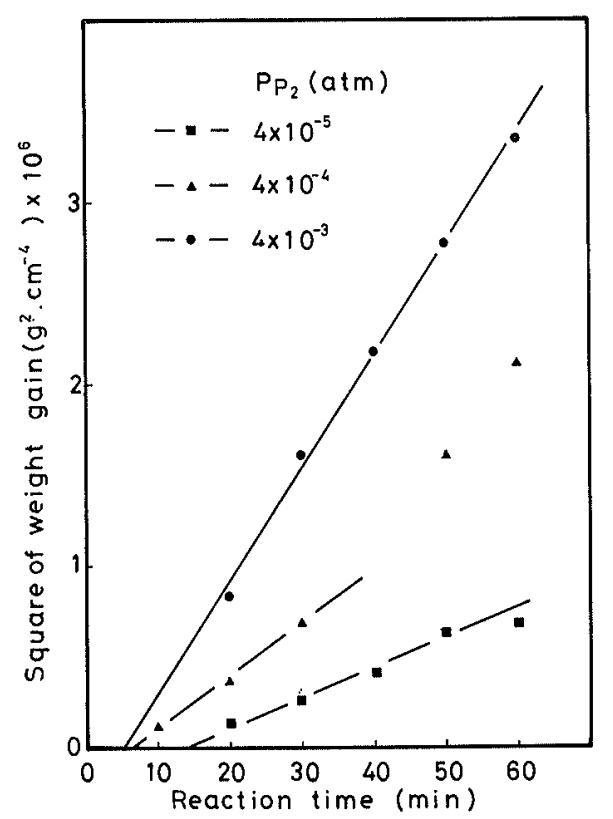

Fig. 6. Parabolic relation between weight gain of the sample and reaction time in the case of the electrolytic iron at $1050^{\circ} \mathrm{C}$. 


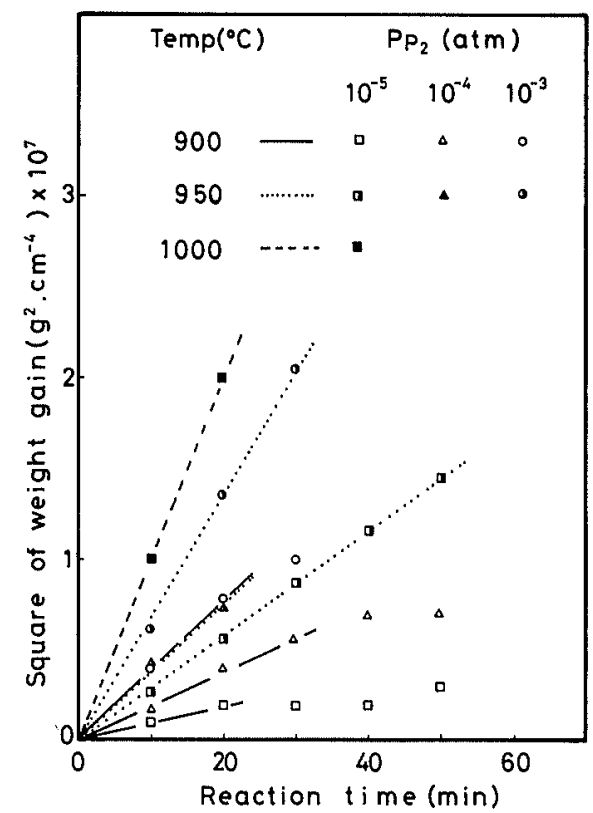

Fig. 7. Parabolic relation between weight gain of the sample and reaction time in the case of the electrolytic iron at 900,950 and $1000^{\circ} \mathrm{C}$.

Table 4. Apparent rate constants of parabolic equation in the case of the killed steel sheet. $\left(\mathrm{g}^{2} \cdot \mathrm{cm}^{-4} \cdot \mathrm{min}^{-1}\right)$

\begin{tabular}{cccc}
\hline \multirow{2}{*}{$\begin{array}{c}\text { Temperature } \\
\left({ }^{\circ} \mathrm{C}\right)\end{array}$} & \multicolumn{3}{c}{ Phosphorus pressure (atm) } \\
\cline { 2 - 4 } & $4 \times 10^{-6}$ & $4 \times 10^{-4}$ & $4 \times 10^{-2}$ \\
\hline 950 & $1.1 \times 10^{-8}$ & $3.1 \times 10^{-8}$ & $5.3 \times 10^{-8}$ \\
1000 & $3.6 \times 10^{-8}$ & $9.8 \times 10^{-8}$ & $1.3 \times 10^{-7}$ \\
1030 & $5.9 \times 10^{-8}$ & & $2.5 \times 10^{-7}$ \\
\hline
\end{tabular}

Table 5. Apparent rate constants of parabolic equation in the case of the electrolytic iron. $\left(\mathrm{g}^{2} \cdot \mathrm{cm}^{-4} \cdot \mathrm{min}^{-1}\right)$

\begin{tabular}{cccc}
\hline \multirow{2}{*}{$\begin{array}{c}\text { Temperature } \\
\left({ }^{\circ} \mathrm{C}\right)\end{array}$} & \multicolumn{3}{c}{ Phosphorus pressure (atm) } \\
\cline { 2 - 4 } & $4 \times 10^{-5}$ & $4 \times 10^{-4}$ & $4 \times 10^{-8}$ \\
\hline 900 & $1.0 \times 10^{-9}$ & $1.9 \times 10^{-9}$ & $3.9 \times 10^{-9}$ \\
950 & $2.9 \times 10^{-9}$ & $3.9 \times 10^{-9}$ & $6.8 \times 10^{-9}$ \\
1000 & $1.0 \times 10^{-8}$ & & \\
1050 & $2.0 \times 10^{-8}$ & $4.3 \times 10^{-8}$ & $5.8 \times 10^{-8}$ \\
\hline
\end{tabular}

some in the figure. The slopes of the straight lines in Figs. 5 to 7 are listed in Tables 4 and 5. The slopes are called as an apparent rate constant. The tables show that the apparent rate constants for the steel sheet are about one order larger than that of the electrolytic iron. According to an observation of cross section of the specimens after reaction by SEM, no difference between steel and iron sheets was observed. It is estimated on the basis of the above observation that the difference between these rate constants was due to an accelerative rate which was not observed because of the batch method.

Relation between the apparent rate constants and reciprocal temperature is shown in Fig. 8. Apparent activation energy is $71 \mathrm{kcal} / \mathrm{mol}$ for each line. Regression equations for each line are shown in Table 6.

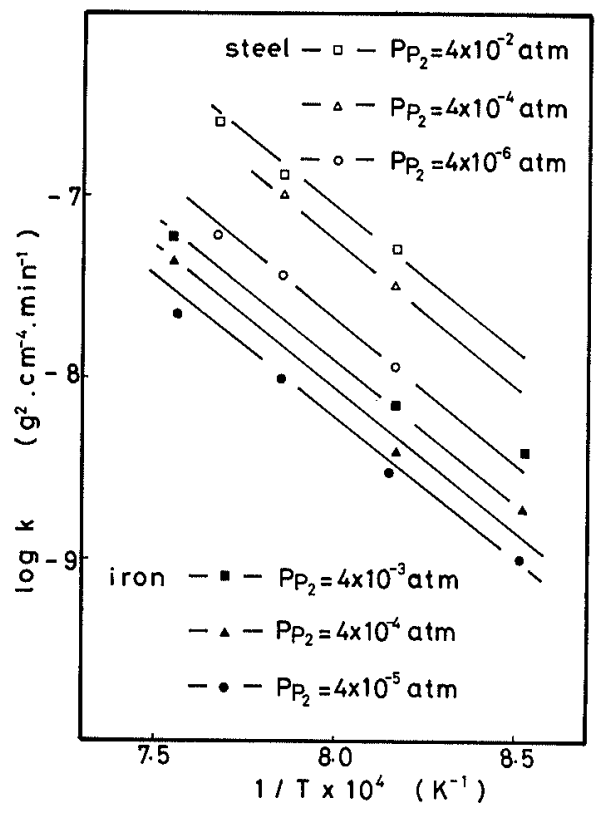

Fig. 8, Relation between logarithm of apparent rate constant and reciprocal temperature.

Table 6. Temperature dependency of apparent rate constants.

$R=1.98 \mathrm{cal} \cdot \mathrm{mol}^{-1} \cdot \mathrm{deg}^{-1}, k: \mathrm{g}^{2} \cdot \mathrm{cm}^{-4} \cdot \mathrm{min}^{-1}$

\begin{tabular}{ccc}
\hline$P_{\mathrm{P}_{2}}(\mathrm{~atm})$ & Sample & Regression equation \\
\hline $4 \times 10^{-6}$ & Steel & $\log k=4.82-71 \times 10^{3} / R T$ \\
$4 \times 10^{-5}$ & Iron & $\log k=4.30-71 \times 10^{3} / R T$ \\
$4 \times 10^{-4}$ & Steel & $\log k=5.25-71 \times 10^{3} / R T$ \\
$4 \times 10^{-4}$ & Iron & $\log k=4.46-71 \times 10^{3} / R T$ \\
$4 \times 10^{-3}$ & Iron & $\log k=4.60-71 \times 10^{3} / R T$ \\
$4 \times 10^{-2}$ & Steel & $\log k=5.45-71 \times 10^{3} / R T$ \\
\hline
\end{tabular}

Iron : Non worked electrolytic iron

Steel : Rolled killed steel

Relation between preexponential terms in Fig. 8 and partial pressure of the phosphorus vapor is shown in Fig. 9. Relation between logarithms of both values is a linear relation. Regression equations of the relation are shown in Table 7 . The apparent rate constants are directly proportional to the $1 / 7$ th power of the phosphorus partial pressure.

On the basis of the Tables 6 and 7 relations between apparent rate constant, partial pressure of phosphorus and temperature are expressed as follows: In the case of the steel sheet,

$$
\log k=0.14 \log P_{\mathrm{P}_{2}}+5.68-15.6 \times 10^{3} / T
$$

In the case of the electrolytic iron,

$$
\log k=0.14 \log P_{\mathrm{P}_{2}}+4.94-15.6 \times 10^{3} / T
$$

Units of $k, P_{\mathrm{P}_{2}}$ and $T$ are $\mathrm{g}^{2} \cdot \mathrm{cm}^{-4} \cdot \mathrm{min}^{-1}$, atm and Kelvin, respectively.

\subsection{EPMA Analysis and $X$-ray Diffraction of Reaction Product}

Cross section of the specimen was observed by EPMA as shown in Fig. 10. A reaction product layer adhering at the surface of the specimen and a 


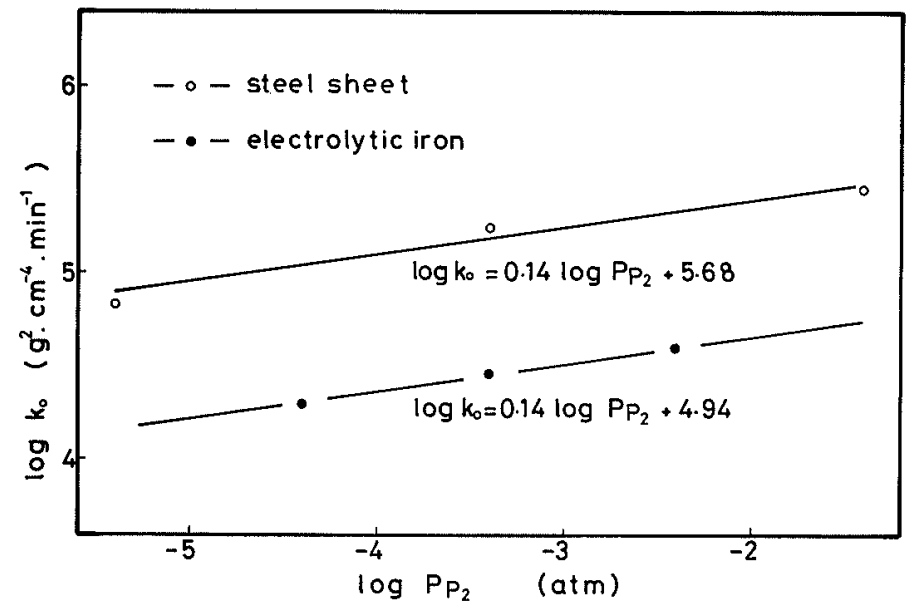

Fig. 9. Relation between logarithm of phosphorus partial pressure and pre-exponential term of the apparent rate constant, $k_{0}$.

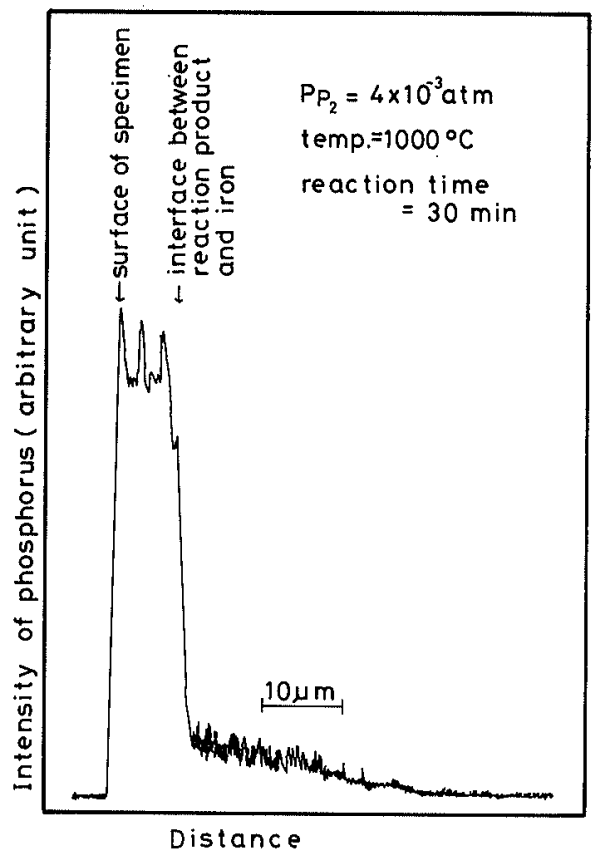

Fig. 10. An example of distribution of phosphorus concentration in the specimen by EPMA analysis.

diffusion layer of phosphorus in a matrix are observed. It was confirmed by X-ray diffraction of Debye-Scherrer method that the reaction product layer was consisted of $\mathrm{Fe}_{2} \mathrm{P}$ as shown in Fig. 11 .

Ratio of the maximum peak height of EPMA analysis in the matrix to that in the reaction product layer is about $1 / 10$. It can be estimated on the basis of the peak height ratio and 21.6 mass $\%$ of concentration of phosphorus in $\mathrm{Fe}_{2} \mathrm{P}$ that the maximum concentration of phosphorus in the matrix almost corresponds to a solubility limit of phosphorus in iron, 2.5 mass $\%{ }^{7}$ )

Fig. 12 shows the cross section of view of the specimen after reaction observed by SEM. A clearance between the surface of the specimen and the reaction product can be observed. On the basis of the observation, it is estimated that the stagnant and accelerative rate of weight change observed with the
Table 7. Effect of phosphorus vapor pressure on rate constants.

\begin{tabular}{ll}
\hline Killed and rolled steel & $\log k_{0}=0.14 \log P_{\mathrm{P}_{2}}+5.68$ \\
Electrolytic iron & $\log k_{0}=0.14 \log P_{\mathrm{P}_{2}}+4.94$ \\
\hline
\end{tabular}

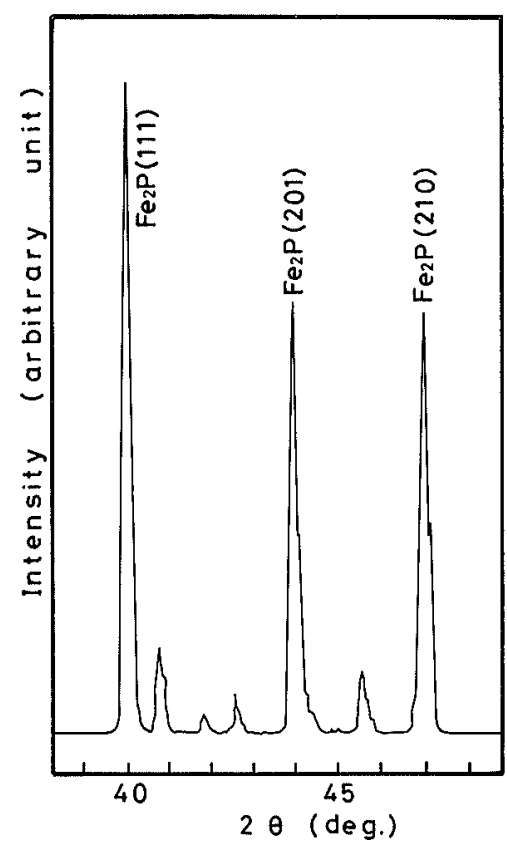

Fig. 11. Diffraction pattern of the reaction product by Debye-Scherrer method.

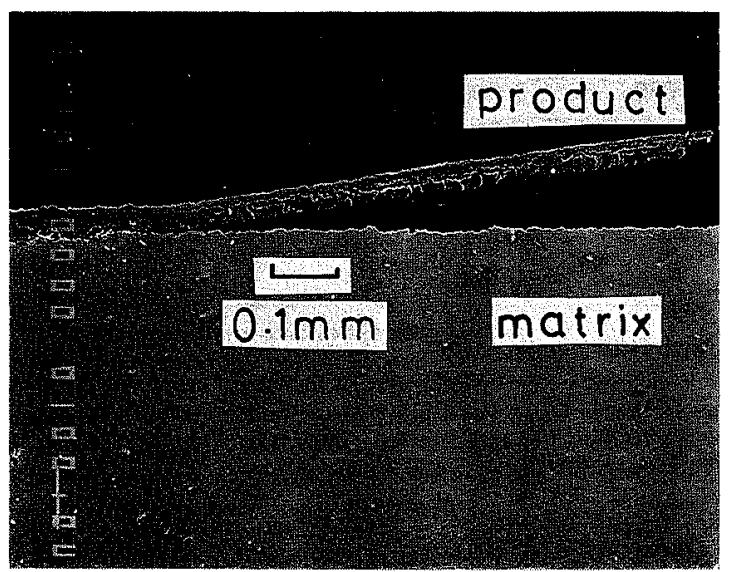

Temperature: $950^{\circ} \mathrm{C}$

$P_{\mathrm{P}_{2}}: 4 \times 10^{-3} \mathrm{~atm}$

Reaction time: $20 \mathrm{~min}$

Fig. 12. Cross section view of an interface between product and iron phases observed by SEM.

continuous method were caused by a formation of voids between the product layer and matrix and by breaks of the layer, respectively, because of stress due to difference in densities, e.g., $6.70 \mathrm{~g} / \mathrm{cm}^{3}$ of reaction product ${ }^{8)}$ and $7.87 \mathrm{~g} / \mathrm{cm}^{3}$ of matrix ${ }^{9)}$ at $20^{\circ} \mathrm{C}$.

\section{Discussion on Rate Determining Step of Reaction}

It is estimated that one of following reaction steps or combined steps is a rate determining step. 
(A) Mass transport in a gas boundary layer

(B) Chemical reaction at an interface between gas and $\mathrm{Fe}_{2} \mathrm{P}$ phases

(C) Mass transport in the $\mathrm{Fe}_{2} \mathrm{P}$ phase

(D) Chemical reaction at an interface between $\mathrm{Fe}_{2} \mathrm{P}$ and solid iron phases, i.e., a formation of $\mathrm{Fe}_{2} \mathrm{P}$

(E) Dissolution of phosphorus into the solid iron

(F) Mass transport in the solid iron

In the case of that one of the steps mentioned above is a rate determining step, (A), (B), (D) and (E) are omitted for the discussion because the rates in the cases do not obey the parabolic rate law.

Relation between weight gain per unit reaction area, $\Delta W$, and thickness of the reaction product, $X$, is expressed as Eq. (4), if the rate determining step is mass transport in the reaction product and concentration of a diffusing material in the reaction product, $\rho^{\prime}$, is nearly constant,

$$
X \rho^{\prime}=\Delta W
$$

When the reaction rate follows a parabolic rate law introduced by Wagner and Grünewald ${ }^{10)}$ a relation between weight gain, $\Delta W$, and reaction time, $t$, can be expressed as Eq. (5),

$$
\left(J_{D}\right)_{X=0}=\left(\frac{d W}{d t}\right)=-D\left(\frac{d C}{d X}\right)_{X=0}=-\rho^{\prime} D\left(\frac{d C}{d W}\right)
$$

where $X=0$ is at an interface between gas and solid phases, and direction to inside of solid is positive sign. From Eq. (5)

$$
\int_{0}^{\Delta W} \int_{0}^{\Delta W} d^{2} W=-\rho^{\prime} D \int_{C_{s}}^{C_{i}} \int_{0}^{t} d C d t
$$

where, $C_{s}, C_{i}$ : concentrations of a diffusing substance at interfaces between gas and reaction product phases as well as between reaction product and solid iron phase, respectively

$D:$ diffusion coefficient.

Eq. (6) can be rewritten as Eq. (7),

$$
\Delta W^{2}=2 \rho^{\prime} D\left(C_{s}-C_{i}\right) t=k_{D} t
$$

where, $k_{D}=2 \rho^{\prime} D\left(C_{s}-C_{i}\right)$.

When it is assumed that the diffusion substance in the reaction product is $\mathrm{P}^{4-}$ ion or $\mathrm{Fe}^{2+}$ ion, relation between $\mathrm{P}_{2}$ in gas phase and a $\mathrm{P}^{4-}$ ion in reaction product phase is follows:

$$
\mathrm{P}_{2}=2 \mathrm{P}^{4-}+8 h^{\circ}
$$

where, $h^{*}$ : a positive hole.

An equilibrium relation of Eq. (8) is expressed as Eq. (9).

$$
\left[\mathrm{P}^{4-}\right]^{2}\left[h^{*}\right]^{8}=K_{p} P_{\mathrm{P}_{z}}
$$

where, $\kappa_{p}:$ an equilibrium constant

[ ]: concentration

$P$ : partial pressure.

When $\left[\mathrm{P}^{4-}\right]$ in a product is nearly constant, the diffusion phenomenon in the product layer can be characterized by the diffusion of the positive hole. When $C_{s}=\left[h^{\circ}\right]_{s}$ and $C_{s} \gg C_{i}$ are assumed, Eq. (10) can be obtained on the basis of Eqs. (7) and (9).

$$
\Delta W^{2}=2 \rho^{\prime} D\left(P_{\mathrm{P}_{2}}\right)^{1 / 8} K_{p}^{\prime} t=k_{D} t
$$

where, $K_{p}^{\prime}:$ a constant

$k_{D}$ : a apparent rate constant.

On the basis of the above discussion, it is expected that $\Delta W^{2}$ and the apparent rate constant, $\kappa_{D}$, are directly proportional to time, $t$, and the $1 / 8$ th power of $P_{\mathrm{P}_{2}}$, respectively.

If the rate determining step is mass transport rate in solid iron phase, the weight gain, $\Delta W$, can be expressed as Eq. (11).

$$
\Delta W=\int_{0}^{t} J_{X=L} d t=-D \int_{0}^{t}\left(\frac{d C}{d X}\right)_{X=L} d t
$$

where, $L:$ a thickness of a production layer

$C:$ a concentration of a diffusion substance $J_{X=L}:$ a flux of a diffusion substance at $X=L$. On the basis of a solution for diffusion in a semiinfinity substance Eq. (12) can be expressed,

$$
\frac{C_{X=L+X^{\prime}}}{C_{X=L}}=1-\frac{2}{\sqrt{\pi}} \int_{L / 2 \sqrt{D t}}^{\left(L+X^{\prime}\right) / 2 \sqrt{D t}} \exp \left(-y^{2}\right) d y
$$

where, $X^{\prime}:$ a distance in the solid iron from the interface between the reaction product and the solid iron $y=\left(L+X^{\prime}\right) / 2 \sqrt{D t}$.

If Eq. (12) is differentiated with respect to $X$, Eq. (13) can be obtained.

$$
(d C / d X)_{X=L}=-C_{X=L} \sqrt{\pi D t}
$$

On the basis of the Eqs. (11) and (13), Eq. (14) can be obtained,

$$
\Delta W=2 C_{X=L} \sqrt{D t / \pi}=K_{B} \sqrt{t}
$$

where, $\quad K_{B}:$ a constant if $C_{X=L}$ is a constant

$$
C_{X=L}: \text { a solubility limit of phosphorus into }
$$
solid iron.

On the basis of the above discussion it is estimated that the relation between $\Delta W$ and $\sqrt{t}$ is a linear relation.

Because the experimental results show that the apparent rate constant is direct proportion to the $1 / 7$ th power of phosphorus partial pressure and an activation energy of diffusion of phosphorus in alpha or gamma phase iron is 45 to $55 \mathrm{kcal} / \mathrm{mol}$ by a literature, ${ }^{11}$ it is estimated that diffusion step in the iron phase is not the rate determining step.

It is concluded that the rate determining step is the diffusion rate in the product layer.

\section{Summary}

In the experiments in which solid iron was reacted with phosphorus vapor at temperature between 900 and $1050^{\circ} \mathrm{C}$, the following things are observed:

(1) The reaction rate can be expressed as a parabolic rate law. 
(2) Apparent rate constants are direct proportion to the $1 / 7$ th power of phosphorus partial pressure.

(3) Reaction product was $\mathrm{Fe}_{2} \mathrm{P}$.

The authors wish deeply to thank students of Chiba Institute of Technology, Mr. K. Okano (now Chiba Civil Corporation), Mr. K. Mitsui (now Japan CMK Co. Ltd.), Mr. E. Tabata (now Izawa Metal Go. Ltd.), Mr. H. Oowada (now Hitachi Automobile Engineering Co. Ltd.), Mr. A. Tanaka (now Powder Tech. Co. Ltd.), Mr. T. Yabe (now Fuji Electrochemistry Co. Ltd.) and Mr. H. Kato (now Nippon Yakin Kogyo Co. Ltd.) for their experimental works, Professor M. Maeda and Mr. T. Ikeda of Institute of Industrial Science, The University of Tokyo for their kind guidance on electro-thermobalance and The Iron and Steel Institute of Japan for its financial support.

\section{REFERENCES}

1) G. H. Herty, Jr.: Trans. Am. Inst. Min., Metall. Pet. Eng., 73 (1926), 1107.
2) W. Oelsen and H. Maetz: Stahl Eisen, 69 (1949), 147.

3) R. Jon, J. Vigliengo, J. Michard, R. Scimar, R. Vidal, A. Poos and A. Decker: Metall. Rep. C.N.R.M., (1968), No. $15,3$.

4) K. Sasa and T. Cho: Tetsu-to-Hagané, 69 (1983), 27.

5) I. Barin, O. Knacke and O. Kubaschevski: Thermochemical Properties of Inorganic Substances, Springer-Verlag, Berlin, (1973), 116, 162, 690, 1182, and supplement p. 110 and 505.

6) I. Barin, O. Knacke and O. Kubaschevski: Thermochemical Properties of Inorganic Substances, Springer-Verlag, Berlin, (1973), 591, 592.

7) Tekko Binran, 3rd ed., Vol. 1, ed. by ISIJ, Maruzen, Tokyo, (1981), 419.

8) S. Hatano: Kogyo Zairyo Binran, Nikkan Kogyo Shinbunsha, Tokyo, (1988), 578.

9) Rikagaku Jiten, ed. by B. Inouye, M. Kotani and B. Tamamushi, Iwanami-Shoten, Tokyo, (1958), 892.

10) G. Wagner and K. Grünewald: Z. Phys. Chem., B., 40 (1938), 455.

11) Tekko Binran, 3rd ed., Vol. 1, ed. by ISIJ, Maruzen, Tokyo, (1981), 351. 